\title{
ESTRATEGIAS PARA LA CONSERVACIÓN DE LA POBLACIÓN DE Pinus hartwegii Lindl. EN LA REGIÓN DEL PEROTE, VERACRUZ
}

\section{Lourdes G. Iglesias Andreu, Juan Alba Landa, Jorge L. Enriquez}

\section{INTRODUCCIÓN}

Los pinos se encuentran entre los organismos genéticamente más diversos. Su sistema genético, que favorece la creación y recombinación de la variación genética, les ha permitido evolucionar en concierto con los cambios ambientales, tanto espaciales como temporales, a los que han estado sometidos desde que iniciaron su divergencia de su forma ancestral, hace poco más de 200 millones de años (Ledig, 1998). Sin embargo esta diversidad se ha visto, sobre todo en los últimos años, seriamente amenazada, debido entre otros a la alta tasa de deforestación que se ha ido produciendo. Se estima que anualmente se deforestan alrededor de 212 mil hectáreas por diversas causas, entre ellas por: desmonte, incendios forestales, cambios del uso de suelo, plagas y enfermedades y aprovechamiento ilegal (Hernández, 1997). Todo ello ha conllevado a que en México - considerado como Centro Secundario de especiación de los pinos- ocupe el cuarto lugar por presentar una elevada tasa anual de deforestación (Ledig, 1998).

Este aspecto cobra una connotación especial si se tiene en cuenta que gran parte de este rico patrimonio, constituido en muchos casos por poblaciones genéticamente únicas, se encuentra en serio peligro de extinción (Ledig, 1997). De acuerdo con este autor el $17 \%$ de las especies vegetales mexicanas se encuentra en peligro de extinción.
Dentro de esto, el peligro más grave lo constituye la pérdida de poblaciones y recursos genéticos dentro de especies, lo que Ledig (1993) ha dado en llamar las «extinciones ocultas».

La pérdida de la diversidad genética, o inclusive los cambios en la estructura genética sin una aparente disminución de la diversidad, podrían conllevar a cambios en el flujo de energía y nutrientes en el ecosistema (Ledig, 1991). Además la diversidad genética, como apuntara Loo (1997), es necesaria para contrarrestar el riesgo de la vulnerabilidad a plagas y factores adversos del ambiente, preservar los complejos de genes adaptados, que pueden ser importantes para la supervivencia de poblaciones viables adaptadas a sus ambientes así como para garantizar la estabilidad de ecosistemas completos.

En México, al igual que en muchos países de la región de Centro América y el Caribe, las poblaciones correspondientes a las más bajas elevaciones (menores de $500 \mathrm{msnm}$ ) son las que resultan en general más vulnerables (Ledig, 1997). No obstante no escapan a la erosión genética las poblaciones de especies que, como la de Pinus hartwegii Lindl., se desarrollan a más altas elevaciones (3500-4000 msnm).

En el Parque Nacional Cofre de Perote se encuentra entre los 3500-4000 msnm, un bosque de Pinus hartwegii Lindl. poco denso y monoespecífico en su estrato arbóreo, cuyas semillas han perdido notablemente su viabilidad de modo 


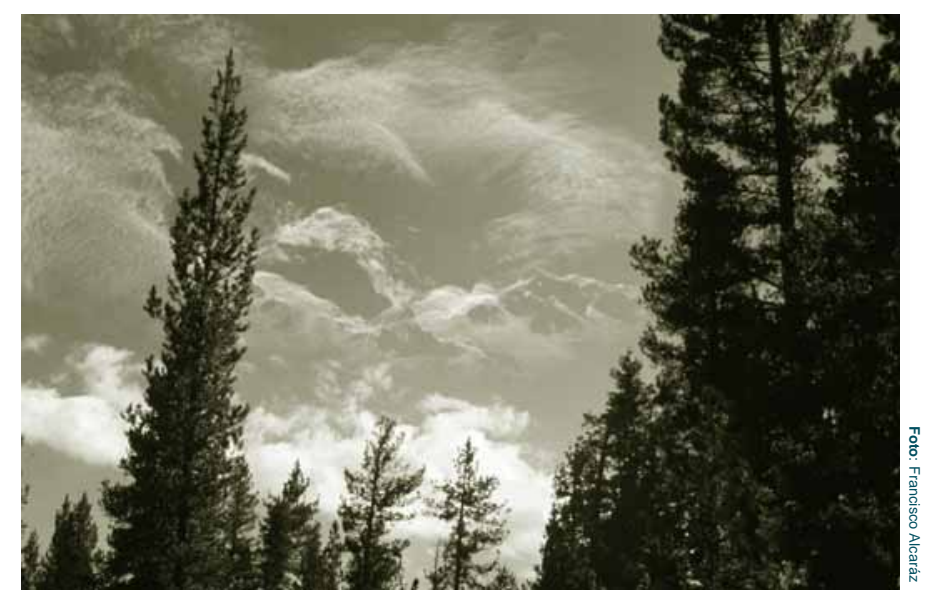

Paisaje de pinos de San Pedro Mártir, México.

que se ha detectado un porcentaje muy bajo (menor de un 10\%) de germinación (Iglesias, 1999, datos no publicados).

La irradiación con rayos $\mathrm{X}$ a muestras de semillas de esta población ha revelado la existencia de una alta carga genética, medida como «Equivalentes de Letalidad Embrionaria», que sobrepasa la cifra del 50\% (Iglesias et al., 1999, datos no publicados). Esto está ocasionando una sensible disminución en la producción y calidad de la semilla de esta población de pinos. Esta respuesta pudiera atribuirse a la acumulación de alelos recesivos letales o genes deletéreos recesivos — que pueden llegar a ocasionar la muerte selectiva de los embriones_-, motivada por una alta tasa de cruzamientos consanguíneos en dicha población.

Como se sabe el sistema de apareamiento de los pinos es flexible para facilitar la invasión a nuevos hábitats, por lo que a pesar de que el patrón predominante de apareamiento en los pinos es la alogamia pueden darse cruzamientos consanguíneos en mayor o menor grado (Ledig, 1998). De este modo, cuando se presentan elevadas tasas de cruzamientos consanguíneos en una población se produce el fenómeno de la depresión consanguínea - como consecuencia de incrementos en la carga de alelos letales, manifestados como genes recesivos deletéreosque pueden ocasionar una reducción del vigor y las tasas reproductivas en dicha población (Williams y Savolaienen, 1996).
Para prevenir los efectos de la depresión consanguínea los pinos cuentan con efectivas barreras postcigóticas, que permiten reducir la oportunidad de que los cigotos derivados de cruzamientos consanguíneos sobrevivan y se reproduzcan (Ledig, 1998). Es así como, a través del análisis de la carga genética mutacional, se puede explicar la necesidad de mantener elevados niveles de diversidad genética en los pinos y comprender por qué el sistema de apareamiento de los pinos resulta eminentemente alógamo.

Al igual que Pinus hartwegii Lindl., otras poblaciones de pinos pueden estar sufriendo los desfavorables efectos de la depresión consanguínea.

\section{PROPUESTA PARA EL ESTABLE- CIMIENTO DE UNA ESTRATEGIA BIOTECNOLÓGICA DE CONSERVACIÓN DE LA POBLACIÓN DE Pinus hartwegii Lindl. DEL COFRE DE PEROTE}

Para establecer una estrategia biotecnológica efectiva de conservación en la población antes mencionada de Pinus hartwegii Lindl. es preciso tener en cuenta, entre otras, la necesidad de:

- Desarrollar nuevas tecnologías para la conservación por vía biotecnológica, ya sea a medio plazo por lento crecimiento o a más largo plazo por crioconservación, de meristemos, polen y otros tejidos vegetativos de esta especie. Aunque los bancos de semillas constituyen la vía que 
más se emplea para la conservación ex situ de los recursos forestales (Ledig, 1997), el empleo de las técnicas de conservación in vitro puede llegar a constituir una estrategia complementaria para la conservación de recursos forestales (Wang et al., 1993, citado por Wang y Beardmore, 1997). Como indicaran Wang y Beardmore (1997), los cultivos in vitro pueden conservarse a corto (1 semana a 2 meses), medio (2 meses a 2 años) y largo plazo (más de 2 años) mediante las técnicas de lento crecimiento y crioconservación. Aunque se requiere efectuar más investigación para desarrollar combinaciones óptimas de crioprotectores y procedimientos de evaluación para reforzar la recuperación después del crioalmacenamiento, diversos autores han logrado avances en esta dirección, sobre todo en especies de coníferas (Monod et al., 1992). Considerando todo lo antes expuesto sería de gran utilidad establecer la técnica para la conservación por lento crecimiento a partir de meristemos apicales de genotipos de la mencionada población de $P$. hartweggii Lindl.

- Obtener estimados precisos de la diversidad genética verdaderamente disponible en esta población mediante el desarrollo de ensayos clásicos de procedencias y progenies y el uso de técnicas moleculares. La importancia de estos estudios para describir y medir la diversidad existente con vistas a lograr una mejor comprensión de los procesos genéticos que operan a nivel poblacional en las especies forestales ha sido puesta de relieve, entre otros, por Bermejo (1997), Parker et al. (1997), Vendramin (1998), Iglesias et al. (1998), Iglesias y García (1999) y Delgado et al. (1999), empleando diversos tipos de marcadores genéticos. Sobre esta base hoy ya se cuenta con resultados preliminares sobre variaciones en las características morfométricas y de actividad enzimática (peroxidasas y amilasas) en semillas de esta población (Iglesias y García, 1999a). Se espera que pueda contarse próximamente con nuevos marcadores tanto isoenzimáticos (esterasas, fosfatasas ácidas) como moleculares (polimorfismo de ADN amplificado al azar, RAPD), que permitan:

- Obtener estimados del grado de diversidad genética aún disponible en dicha población.

- Monitorear la estabilidad genética del material micropropagado o conservado in vitro.
- Contribuir a la selección de árboles superiores al promedio en dicha población.

- Asistir al programa de reforestación garantizando por esta vía la pureza genética del material a replantar y reducir con ello el uso de fuentes de semillas no adaptadas así como contaminaciones locales de estos recursos.

- Proporcionar información valiosa sobre la estructura genética de la población, su historia evolutiva reciente y sus relaciones genéticas con otras poblaciones aledañas.

- Utilización de diversas técnicas biotecnológicas: variación somaclonal, gametoclonal y protoclonal, que permitan ampliar la base genética en dicha población. La aplicación de las mencionadas técnicas permitiría la selección de genotipos elites y establecer un mosaico de variantes genéticas en ésta población. Esta vía biotecnológica se ha extendido como un método alternativo para acelerar los trabajos de mejora forestal, ya que induce cambios genéticos en el material cultivado in vitro. Algunos de estos cambios se manifiestan como alteraciones en el número cromosómico y diversas aberraciones cromosómicas, en variaciones relacionadas con la resistencia a enfermedades, tolerancia a estrés abiótico, así como otros cambios en la composición de proteínas de las semillas (Brettel e Ingram, 1979; Flinn et al., 1991).

- Fomentar el uso de las técnicas nucleares en combinación o no con las técnicas de cultivo de tejidos. En la actualidad se cuenta con una voluminosa información de la aplicación de las técnicas nucleares con diversos propósitos en especies de pinos. Algunos autores (Rudolph, 1979; Sokolov et al., 1998) han resaltado la utilidad del empleo de bajas radiaciones de rayos $\mathrm{X}$, para evaluar el porcentaje de semillas con letalidad embrionaria, así como el uso de bajas dosis de radiaciones gamma para modificar las características de viabilidad de la semilla. En el Centro de Genética Forestal se están iniciando los trabajos para que, en colaboración con la Facultad de Instrumentación y Electrónica de la Universidad Veracruzana y el Hospital Macuiltepec, se lleven a cabo estos trabajos en esta población de Pinus hartwegii Lindl. El propósito fundamental es encontrar técnicas rápidas y no destructivas para monitorear el número de Equivalentes de Letalidad 


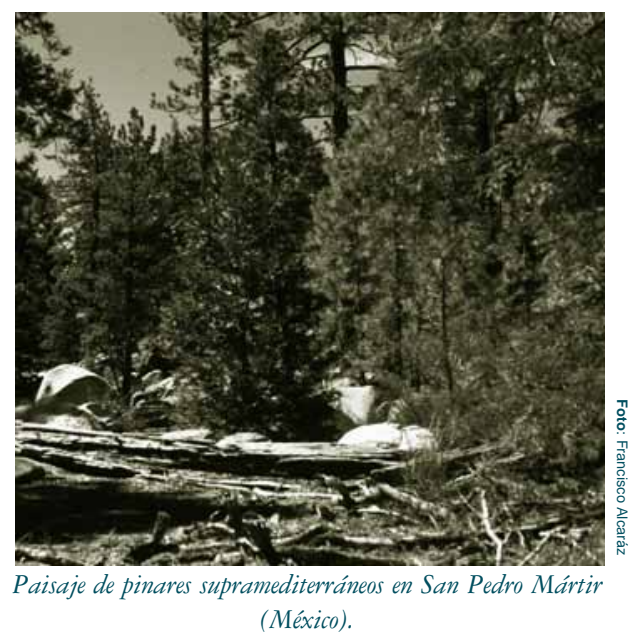

Embrionaria mediante la aplicación de técnicas como rayos $\mathrm{X}$, e implementar el uso de bajas dosis de radiaciones gamma para modificar las características de viabilidad de la semilla en dicha población.

- Incorporar los avances obtenidos en la aplicación de las técnicas de micropropagación a los programas de reforestación y mejoramiento que se realicen con este objetivo en esta población. En pinos se cuenta con una voluminosa literatura sobre el uso de las técnicas de cultivo de tejidos con propósito de micropropagación, ya sea a través de los procesos de organogénesis como de embriogénesis somática (Tautorus et al., 1991; Sens et al., 1993; Halos y Go, 1993; Magallanes-Cedeño, 1997). De hecho la micropropagación constituye quizás una de las aplicaciones biotecnológicas de mayor importancia para lograr la producción de grandes volúmenes de propágulos elite, libres de enfermedades, que demandan los programas de reforestación intensiva. En el Centro de Genética Forestal se trabaja actualmente estableciendo las técnicas de micropropagación por la vía organogenética para la multiplicación acelerada de Pinus patula Schl. y Cham. Considerando la escasa reproducción en vivero que por todo lo antes expuesto posee la población de Pinus hartwegii Lindl., la utilización de esta técnica biotecnológica pudiera constituir una alternativa muy promisoria para apoyar los programas de reforestación que se desarrollan en esta especie.

\section{CONCLUSIONES}

La estrategia biotecnológica antes propuesta para la población de Pinus hartwegii Lindl. en la región del Perote puede resultar demasiado ambiciosa por la diversidad y multiplicidad de acciones que se proponen. No obstante, es preciso resaltar que muchas de estas propuestas se encuentran ya en ejecución en el Centro de Genética Forestal de la Universidad Veracruzana. Los resultados que de los mismos se obtengan pudieran ser extrapolables desde un punto de vista metodológico a otras especies de Pinus que presenten una problemática similar. Todo ello coadyuvaría a elevar la tasa de reproducción, el vigor y la productividad en muchas de estas poblaciones así como a conservar saludable el ecosistema, que es un objetivo prioritario en el ámbito forestal.

\section{BIBLIOGRAFIA}

Bermejo, B. 1997. Análisis de la Estructura en Poblaciones de Árboles Forestales. En: J.J. Vargas, B. Bermejo y F.Th. Ledig (eds.). Manejo de Recursos Genéticos Forestales. Pp: 37-48.

Brettel, R.I. e Ingram, D.S. 1979. Tissue culture in the production of novel disease resistant crop plants. Biol. Rev. 54:329.

Delgado, P., Piñero, D., Chaos, A., Pérez-Nasser, N. y Álvarez-Byulla, E.R. 1999. High population differentiation and genetic variation in the 
endangered mexican pine Pinus rzedowskii (Pinaceae). Am.J.Bot. 86:669.

Flinn, B. S., Roberts, D.R. y Taylor, I.E.P. 1991. Evaluation of somatic embryos of interior spruce. Characterization and development regulation of storage proteins. Physiol. Plant. 82: 624-632.

Halos, S.C. y Go, N.E. 1993. Micropropagation of Pinus caribaea Morelet. Plant Cell Tiss. Org. Cult. 32:4753.

Hernández, H. 1997. Programa Nacional de Reforestación: Estrategias de corto y mediano plazo (1995-2000). En: J.J. Vargas, B. Bermejo y F.Th. Ledig (eds.). Manejo de Recursos Genéticos Forestales. Pp: 241-252.

Iglesias, L., García, J. y Aparicio, A. 1998. Estudio del polimorfismo proteico en Pinus greggii Engelm en Veracruz, México. Nota Técnica No. 45. $6 p p$.

Iglesias, L. y García, J. 1999. Principales avances en el estudio de la diversidad genética en árboles mediante el uso de marcadores moleculares. Foresta Veracruzana 1 (2): 51-56.

Iglesias, L. y García, J. 1999a. Variabilidad morfométrica y bioquímica en semillas de especies de Pinus provenientes de la Región del Cofre de Perote, Veracruz. (En vías de publicación).

Ledig, F.Th. 1991. The role of genetic diversity in maintaining the global ecosystem, En: Proceedings of the Tenth World Forestry Congress, Paris, Vol. 2. Revue Forestiere Francaise, Nancy, France. Pp: 71-78.

1993. Secret extinctions: The loss of genetic diversity in forest ecosystems, In: M.A. Fenger, E.H. Miller, J.A. Johnosn, E.J.R. Williams (eds.), Our living legacy: Proceedings of a symposium on biological diversity. Royal British Columbia Museum, Victoria, British Columbia. Pp: 127-140.

1997. Conservación y Manejo de Recursos Genéticos Forestales. En: J.J. Vargas, B. Bermejo y F.Th. Ledig (eds.). Manejo de Recursos Genéticos Forestales. Pp: 1-21.

1998. Genetic variation in Pinus.

En: Richardson, D. M. (ed.) Ecology and Biogeography of Pinus. Cambridge University Press. Cambridge.
Loo, J. 1997. Manejo de germoplasma en programas de mejoramiento de árboles. En: J.J. Vargas, B. Bermejo y F.Th. Ledig (eds.). Manejo de Recursos Genéticos Forestales. Pp: 49-65.

Magallanes-Cedeño, M.E. 1997. Aplicación de la tecnología del cultivo in vitro en la propagación de especies forestales. En: J.J. Vargas, B. Bermejo y F.Th. Ledig (eds.). Manejo de Recursos Genéticos Forestales. Pp: 229-240.

Monod, V., Poissonnier, M., Dereuddre, J. y Pasques, M. 1992. Successful cryopreservation of Eucalyptus gunnii shoot-tips in liquid nitrogen. En: Proceedings: Mass production technology for genetically improved fast growing forest tree species, 1418 September 1992, Bordeaux-APOCEL, Nangis. Pp. 135-145.

Parker, K.C., Hamrick, J.L., Parker, A.J. y Stacy, E.A. 1997. Allozyme diversity in Pinus virginiana (Pinaceae) intraspecific and interspecific comparisons. Am.J.Bot. 84(10): 1372-1382.

Rudolph, T. D. 1979. Effects of gamma irradiation of Pinus banksiana Lamb. seed as expressed by $\mathrm{M}$ 1 trees over 10-year period. Env. Exp. Bot. 19(2): 85-92.

Sen, S., Aimers-Halliday, J., McKinley, C. R. y Newton, R. J. 1993. Micropropagation of conifers by organogenesis. Plant Physiol. 12: 129135.

Sokolov, M. V., Isayenkov, S. V. y Sorochynskyi, B. V. 1998. Low-dose irradiation can modify viability characteritics of common pine (Pinus sylvestris) seeds. Tsitologiya ; Genetika 32(4): 6571.

Tautorus, T.E., Fowke, L.C y Dunstan, D.1. 1991. Somatic embryogenesis in conifers. Can.J.Bot. 69: 1873-1889.

Vendramin, G.G., Anzidei, M., Madghiele, A. y Bucci, G. 1998. Distribution of genetic diversity in Pinus pinaster Ait. as revealed by chloroplast microsatellites. T.I.G. 97: 456-463.

Wang, B.S.P. y Beardmore, T. 1997. Almacenamiento y manejo de germoplasma. En: J.J. Vargas, B. Bermejo y F.Th. Ledig (eds.). Manejo de Recursos Genéticos Forestales. Pp: P107140.

Williams, S.C.G. y Savolainen, O. 1996. Inbreeding depresion in conifers. Forest Sci. 41(2): 102-117. 